\title{
Hemifusion in Synaptic Vesicle Cycle
}

\author{
Dae-Hyuk Kweon ${ }^{1}$, Byoungjae Kong ${ }^{1}$ and Yeon-Kyun Shin ${ }^{2 *}$ \\ ${ }^{1}$ Department of Genetic Engineering, College of Biotechnology and Bioengineering, Sungkyunkwan University, Suwon, \\ South Korea, ${ }^{2}$ Department of Biochemistry, Biophysics and Molecular Biology, lowa State University, Ames, IA, USA
}

In the neuron, early neurotransmitters are released through the fusion pore prior to the complete vesicle fusion. It has been thought that the fusion pore is a gap junction-like structure made of transmembrane domains (TMDs) of soluble N-ethylmaleimidesensitive-factor attachment protein receptor (SNARE) proteins. However, evidence has accumulated that lipid mixing occurs prior to the neurotransmitter release through the fusion pore lined predominantly with lipids. To explain these observations, the hemifusion, a membrane structure in which two bilayers are partially merged, has emerged as a key step preceding the formation of the fusion pore. Furthermore, the hemifusion appears to be the bona fide intermediate step not only for the synaptic vesicle cycle, but for a wide range of membrane remodeling processes, such as viral membrane fusion and endocytotic membrane fission.

Keywords: SNARE, membrane fusion, hemifusion, fusion pore, transmembrane domain

\section{INTRODUCTION}

Neurotransmitter release from the neuron requires fusion of vesicles to the plasma membrane. However, the bilayer structure is highly stable; thus, two bilayers normally do not fuse spontaneously. It is thought that conserved soluble $\mathrm{N}$-ethylmaleimide-sensitive-factor attachment protein receptor (SNARE) proteins mediate synaptic vesicle fusion (Söllner et al., 1993a,b). Three SNARE proteins involved in neuroexocytosis are synaptobrevin-2 (Syb2, also called VAMP2),

Edited by:

Cong Ma,

Huazhong University of Science and

Technology, China

Reviewed by: Tabrez Jamal Siddiqui, University of Manitoba, Canada Yuji Ishitsuka, University of Illinois at Urbana-Champaign, USA

${ }^{*}$ Correspondence:

Yeon-Kyun Shin colishin@iastate.edu

Received: 27 December 2016 Accepted: 27 February 2017

Published: 16 March 2017

Citation:

Kweon D-H, Kong B and Shin Y-K (2017) Hemifusion in Synaptic Vesicle Cycle.

Front. Mol. Neurosci. 10:65 doi: 10.3389/fnmol.2017.00065 syntaxin-1 (Stx1), and SNAP-25. Syb2 is the vesicle membrane (v-) SNARE of 116-amino acids with a single transmembrane domain (TMD). Stx1 is the 288 -amino acid protein attached to the plasma membrane, likewise with a single TMD. SNAP-25 has lipid anchors in the plasma membrane and forms the target membrane (t)-SNARE complex with Stx1 (Figure 1A). Cognate SNARE motifs that protrude respectively from two membranes assemble to form a parallel four-helix bundle (Poirier et al., 1998; Sutton et al., 1998) that drives apposition and subsequent fusion of two membranes (Weber et al., 1998). It is believed that SNARE proteins progressively zipper from the membrane-distal N-terminal region toward the membrane-proximal C-terminal region (Fiebig et al., 1999; Chen et al., 2001; Melia et al., 2002; Matos et al., 2003; Sorensen et al., 2006; Ellena et al., 2009; Gao et al., 2012; Lou and Shin, 2016).

A crucial question towards elucidating the pathway of membrane fusion is what happens to the bilayers when two membranes merge. Obviously, our interests are on the role that SNARE TMDs play in fusion because they are located at or near the epicenter of membrane fusion. There are two proposed fusion pathways that predict two very different roles of the TMDs. The first model (Han et al., 2004), which is primarily based on electrophysiological measurements, argues that the TMDs serve as the principal building block of the fusion pore that is considered a bona fide intermediate for synaptic vesicle fusion. In stark contrast, the second model (Xu et al., 2005), largely based on spectroscopic and structural studies, predicts that lipids are major building blocks that build the "hemifusion" and the fusion pore. In the latter, TMDs play a supporting role either as membrane anchors for the soluble SNARE complex or as mechanical levers working at the periphery. 
Although the TMD-lined fusion pore model was developed earlier to explain electrophysiological results, much evidence has now accumulated in favor of membrane fusion through hemifusion for SNARE-dependent vesicle fusion. Furthermore, the hemifusion might be a common intermediate shared by many membrane fusion and fission systems including viral-cell membrane fusion (Melikyan et al., 1995; Chernomordik et al., 1998; Chernomordik and Kozlov, 2003) and endocytotic membrane fission (Antonny et al., 2016).

\section{TMD-LINED FUSION PORE}

There is overwhelming evidence that vesicle fusion transits through the fusion pore (Neher and Marty, 1982; Breckenridge and Almers, 1987; Chow et al., 1992; Alvarez de Toledo et al., 1993; Lollike et al., 1995), a metastable intermediate with a small aqueous opening through two apposed membranes. In this state, the vesicle is expected to remain nearly intact except a pore that continues through the plasma membrane. The size of the fusion pore is estimated to be similar to those of large ion channels. Some small but detectable amounts of neurotransmitters can pass through the fusion pore. Electrophysiological measurements of fusion pore conductance revealed that the fusion-pore diameter remains $<3 \mathrm{~nm}$ and can persist for a few seconds (Albillos et al., 1997).

In an earlier study, no flow of lipids was observed at the stage of the fusion pore formation (Klyachko and Jackson, 2002). Combining the electrophysiological data, one could envision a gap junction-like fusion pore in which SNARE complex formation brings about docking of two hemi-pores made of vand t-SNARE TMDs, respectively, to produce a longer, complete pore that continues through the two membranes (Figure 1B).

Experimental evidence that supports such a protein-lined fusion pore was obtained by amperometric study of Stx 1 mutants (Han et al., 2004). Han et al. (2004) found that Trp mutations at the putative pore-lining residues in the TMD interfere with the release of the neurotransmitters, consistent with the model. Based on the conductance measurements, they modeled a helixlined pore consisting of 5-8 TMDs with a $0.5 \mathrm{~nm}$ pore at the center (Figure 1C).

Although the data qualitatively agree with the model, one caveat is that the impedance of the release due to the Trp mutation is much lower than expected. There is only a $20 \%-30 \%$ decrease in the release with the mutation. In reality, one would expect that 5-8 Trp residues at the same layer in the 0.5 -nm-diameter pore would completely fill the space, which would allow little passage of neurotransmitters through the pore. On the other hand, two membranes are still fully separated in this stage, and the model does not explain how structurally and energetically the two bilayers eventually merge to complete the fusion reaction (Figure 1D; Chernomordik and Kozlov, 2003).

Recently, Cys-scanning experiments showed that V101 and I105 in the TMD of Syb2 might line the fusion pore (Bao et al., 2016). However, the small nanodisc of $\sim 6 \mathrm{~nm}$ diameter used in the Cys-scanning experiments, contained as few as two copies of Syb2. The reality is that two copies of the TMD would not be able to form a TMD-lined pore. Alternatively, an idea that the fusion pore may be both lipidic and proteinaceous was suggested on the basis of an amperometry study of chromaffin cells expressing C-terminal truncation mutants of SNAP-25 (Fang et al., 2008). The layout of TMDs in this model is however purely imaginary with little experimental support.

The early result that there was no lipid flow at the fusion pore stage is an important basis for developing a gap junction-like fusion model. However, it is not unusual to have poor lipid mixing when the protein density is high, as is shown for influenza virus-cell fusion (Chernomordik et al., 1998). Thus, one could argue that limited lipid mixing might not be a sufficient condition for a gap junction-like fusion pore. After all, the TMD might not be a required component to complete membrane fusion. In fact, TMD-less, lipid-anchored SNAREs are sufficient for healthy neurotransmitter release in the neuron (Zhou et al., 2013), vacuole fusion in yeast (Xu et al., 2011), and proteoliposome fusion in vitro (McNew et al., 2000), raising concerns on the validity of the TMD-lined fusion pore model.

\section{HEMIFUSION}

Let us now consider alternative possibilities to the TMD-lined fusion pore. For membrane fusion between influenza virus and red blood cells, Kemble et al. (1994); Melikyan et al. (1995) made a seminal discovery that uncovered a lipiddominant intermediate in membrane fusion (Kemble et al., 1994; Melikyan et al., 1995). The authors found that a GPI-anchored hemagglutinin mutant arrests membrane fusion at the intermediate state in which lipid mixing is allowed while content mixing is not.

The observation by Kemble et al. (1994); Melikyan et al. (1995) appears to be consistent with a theoretical model for membrane fusion, developed for protein-free fusion of two lipid bilayers, on the basis of the lipid-stalk intermediate (Kozlov et al., 1983). We call the half-merged state in which inner leaflets are intact while the outer leaflets are merged the "hemifusion". The predicted hemifusion was later imaged experimentally with $\mathrm{x}$-ray crystallography for the macroscopically aligned, protein-free multi-bilayer (Yang and Huang, 2002).

At hemifusion, lipid mixing may be allowed through outer leaflets (Figure 1E). However, lipid mixing through inner leaflets is also possible because the hemifusion could be in equilibrium with small fusion pores that flicker (Figure 1F; Chanturiya et al., 1997). In some cases, the hemifusion diaphragm can be formed via expansion of the hemifusion into a large area (Hernandez et al., 2012).

The idea that intracellular membrane fusion might transit through a structure of the curved membrane was percolated through the observation that the exogenously added lysolipids impair exocytosis in cells (Chernomordik et al., 1993). It has been thought that the molecular shape of a lipid correlates with the effective spontaneous curvature. While cylindrical phosphatidylcholine forms the almost flat monolayer, cone-shaped phosphatidylethanolamine and diacylglycerol bulge in the direction of the acyl chains and favor the net negative curvature. In contrast, lysolipids such as lysophosphatidylcholine and polyphosphoinositides are inverted cone-shaped molecules 


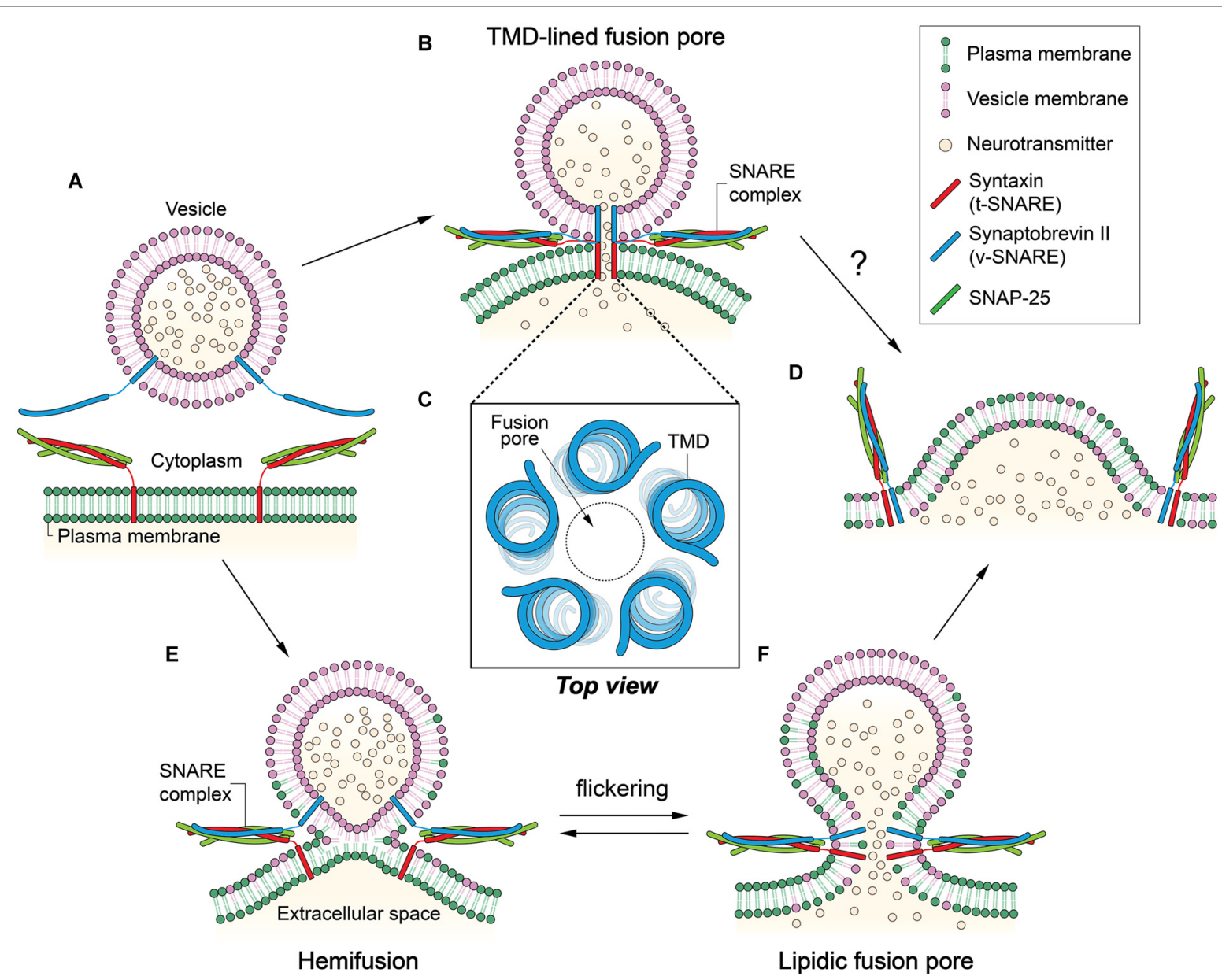

FIGURE 1 | Two contradicting mechanisms for the formation of fusion pore. (A) Synaptobrevin-2 (Syb2) is anchored to a synaptic vesicle, and the t-soluble $\mathrm{N}$-ethylmaleimide-sensitive-factor attachment protein receptor (SNARE) complex composed of syntaxin-1 (Stx1) and SNAP-25 is anchored to the plasma membrane. Membrane fusion is mediated by the formation of the SNARE four-helix bundle. (B) In the transmembrane domain (TMD)-lined fusion pore model, a gap junction-like fusion pore is formed by TMDs of SNARE proteins. Two hemi-pores, one formed by Stx1 TMDs and the other by Syb2 TMDs, dock to constitute a long pore through which neurotransmitters can be released. (C) Five to eight TMDs may form a pore in the center. (D) The TMD-lined pore model cannot explain how fusion between the two membranes is achieved or how the small fusion pore eventually dilates to complete fusion reaction. (E) In the hemifusion, outer leaflets are merged while inner leaflets remain separate. (F) A lipidic fusion pore is formed by inner leaflet mixing. This small fusion pore is in equilibrium with the hemifusion, which may result in flickering. The small fusion pore would eventually dilate to complete membrane fusion.

with large polar heads and thin acyl chain tails that prefer the positively curved membrane to the negatively curved one. Net negative membrane curvature happens to be a characteristic feature of the hemifusion (Chernomordik and Kozlov, 2008).

\section{HEMIFUSION IN SNARE-DEPENDENT MEMBRANE FUSION}

The first direct experimental evidence of hemifusion in SNARE-dependent fusion was from in vitro fusion assays employing SNARE-reconstituted proteoliposomes ( $\mathrm{Lu}$ et al., 2005; Xu et al., 2005). The fluorescence measurements showed that lipids in the outer leaflets mix faster than those in the inner leaflets. Furthermore, the fusion reaction between single proteoliposomes, studied with total internal reflection microscopy, showed distinct steps that reflected the hemifusion (Yoon et al., 2006).

Hemifusion has also been identified in fusion of vacuoles from yeast (Jun and Wickner, 2007) as well as in $\mathrm{Ca}^{2+}$. triggered exocytosis in chromaffin cells (Wong et al., 2007). Additionally, the hemifusion was observed in cell-cell fusion by flipped SNAREs (Giraudo et al., 2006) and in fusion between a SNARE-reconstituted nanodisc and a liposome (Shi et al., 2012), although it is unclear whether the hemifusion observed here is a dead-end product or not. Recently, signals reflecting hemifusion have been detected in the force measurement in SNARE-mediated fusion of proteoliposome to the supported bilayer (Oelkers et al., 2016). Furthermore, a hemifusion structure was visualized in the study of neurons with electron tomography at low resolution (Zampighi et al., 2006). 
Although there is overwhelming evidence that the hemifusion does exist in SNARE-dependent membrane fusion, there is still a controversy concerning whether the hemifusion is on- or off-pathway in $\mathrm{Ca}^{2+}$-triggered exocytosis. In fact, Diao et al. (2012) raised the possibility that hemifusion might be an off-pathway intermediate in $\mathrm{Ca}^{2+}$-triggered exocytosis (Diao et al., 2012). In their in vitro experiments, full fusion has occurred within the pool of un-hemifused proteoliposomes, although it is still possible that the hemifusion is too short-lived to be detected under their experimental conditions. Alternatively, in some limited cases, the hemifusion can expand into the hemifusion diaphragm, which may not progress readily to full fusion (Hernandez et al., 2012).

There is still controversy on whether the TMDs are even required in the neuro-exocytosis. While Zhou et al. (2013) found that GPI-anchored Syb2 fully supports the neurotransmitter release (Zhou et al., 2013). Han et al. (2004) showed that various lipid-anchored Syb2 variants provide little support of exocytosis (Chang et al., 2016). Although SNARE TMDs may not be essential, it turns out that they play important, active roles in modulating SNARE-dependent membrane fusion. For example, Shin et al. (2014) have shown that cholesterol could change the conformation of the dimeric Syb2 TMD to be favorable for membrane fusion (Tong et al., 2009). A simulation study found that the SNARE TMDs might play a role in initiating fusion by distorting the lipid packing of the outer leaflets (Risselada et al., 2011). It is also shown that the conformational flexibility of the Syb2 TMD might lower the negative membrane curvature within the outer leaflet of the fusion pore neck to facilitate pore expansion (Dhara et al., 2016).

\section{COUPLING OF SNARE ZIPPERING TO THE HEMIFUSION}

SNARE motifs assemble into a stable, parallel, four-stranded coiled coil. The SNARE complex is made of 15 (numbered -7 to +8 ) hydrophobic layers and one ionic zeroth layer at the center. There is evidence that SNARE motifs zipper from the membrane-distal $\mathrm{N}$-terminal region towards the membrane-proximal C-terminal region (Fiebig et al., 1999; Chen et al., 2001; Melia et al., 2002; Sorensen et al., 2006; Su et al., 2008; Ellena et al., 2009). After transitioning through intermediate structures that might serve as structural platforms for interactions with other accessory proteins, the SNARE complex ends up at a cis-conformation representing the post fusion state in which TMDs of Stx1 and Syb2 reside in the same membrane. Prior to cis-complex formation, partial complexes are present, of which the degree of zippering was only recently revealed. It was found, from single-molecule force measurements, that SNARE complex formation may occur in at least two steps, with a pivot at the conserved "zeroth" layer in the middle (Li et al., 2007; Gao et al., 2012; Min et al., 2013; Shin et al., 2014; Zorman et al., 2014). Now, given that SNARE zippering drives apposition of two membranes, it must be determined at what stage of SNARE zippering hemifusion occurs. Precise mapping of the degrees of SNARE zippering to specific stages of membrane fusion is prerequisite to the understanding of the mechanism of membrane fusion.

An immediate, related question in the field has been if membranes are hemifused before the $\mathrm{Ca}^{2+}$ influx. Hemifusion induced by SNARE complex formation before $\mathrm{Ca}^{2+}$ was reported (Figure 2B), for the first time, by Schaub et al in their in vitro investigation of the regulation of SNARE-dependent vesicle fusion by Syt1 and complexin (Schaub et al., 2006). This result was verified with the observations that the hemifusion is a stable intermediate of exocytosis in neuronal cells in vivo (Wong et al., 2007) and that two membranes may be hemifused before $\mathrm{Ca}^{2+}$ influx (Zampighi et al., 2006). In contrast, it has been proposed that syt 1 and $\mathrm{Ca}^{2+}$ play a role in driving lipid stalk formation (Martens et al., 2007; Hui et al., 2009), indicating that lipid mixing or stalk formation occur after $\mathrm{Ca}^{2+}$ influx (Figure 2C). However, this model has not been verified with in vivo results.

Interestingly, it has been recently shown that SNARE zippering of only the $\mathrm{N}$-terminal half could drive hemifusion (Figure 2A; Yang et al., 2010). SNARE complex formation can be arrested at the half-zippered state by a flavonoid myricetin, and it is found that the state arrested by myricetin corresponds to the hemifusion in proteoliposome fusion. Myricetin blocks C-terminal zippering by binding to the middle region, while allowing SNARE zippering at the $\mathrm{N}$-terminal region. Remarkably, all hemifused vesicles arrested by myricetin were completely converted to full fusion when the myricetin clamp is removed by an enzyme laccase and the fusion reaction was triggered by $\mathrm{Ca}^{2+}$. The results imply that the hemifusion is likely to be an on-pathway intermediate in $\mathrm{Ca}^{2+}$-triggered exocytosis (Heo et al., 2016). Further, the study raises the strong possibility that the hemifusion is induced by the N-terminal half-zippered SNARE complex.

One might now wonder how hemifusion is possible despite SNAREs being only half-zippered at the N-terminal region. Under these conditions, the SNARE complex would not be forcing apposition of two membranes due to the flexibility at the C-terminal half. Alternatively, the highly basic juxtamembrane regions might play a role here. In fact, electrostatic stitching of two negatively charged membranes by the polybasic sequence has been previously proposed as a potential mechanism for membrane merging (Williams et al., 2009).

Purely energetically speaking, it was recently shown that only one SNARE complex may be sufficient for hemifusion (van den Bogaart et al., 2010; Shi et al., 2012). Partial SNARE zippering generates about $35 k_{\mathrm{B}} T$, corresponding closely to the energy needed for hemifusion (Li et al., 2007). Half-zippering releases $26 k_{\mathrm{B}} T$ in the presence of membranes and $35 k_{\mathrm{B}} T$ in the presence of the pre-assembled C-terminal domains (Gao et al., 2012; Zorman et al., 2014). Because there are multiple SNARE complexes in the synaptic vesicles (Lang et al., 2001; Takamori et al., 2006), it is possible that hemifusion is efficiently achieved by multiple half-zippered SNARE complexes. 


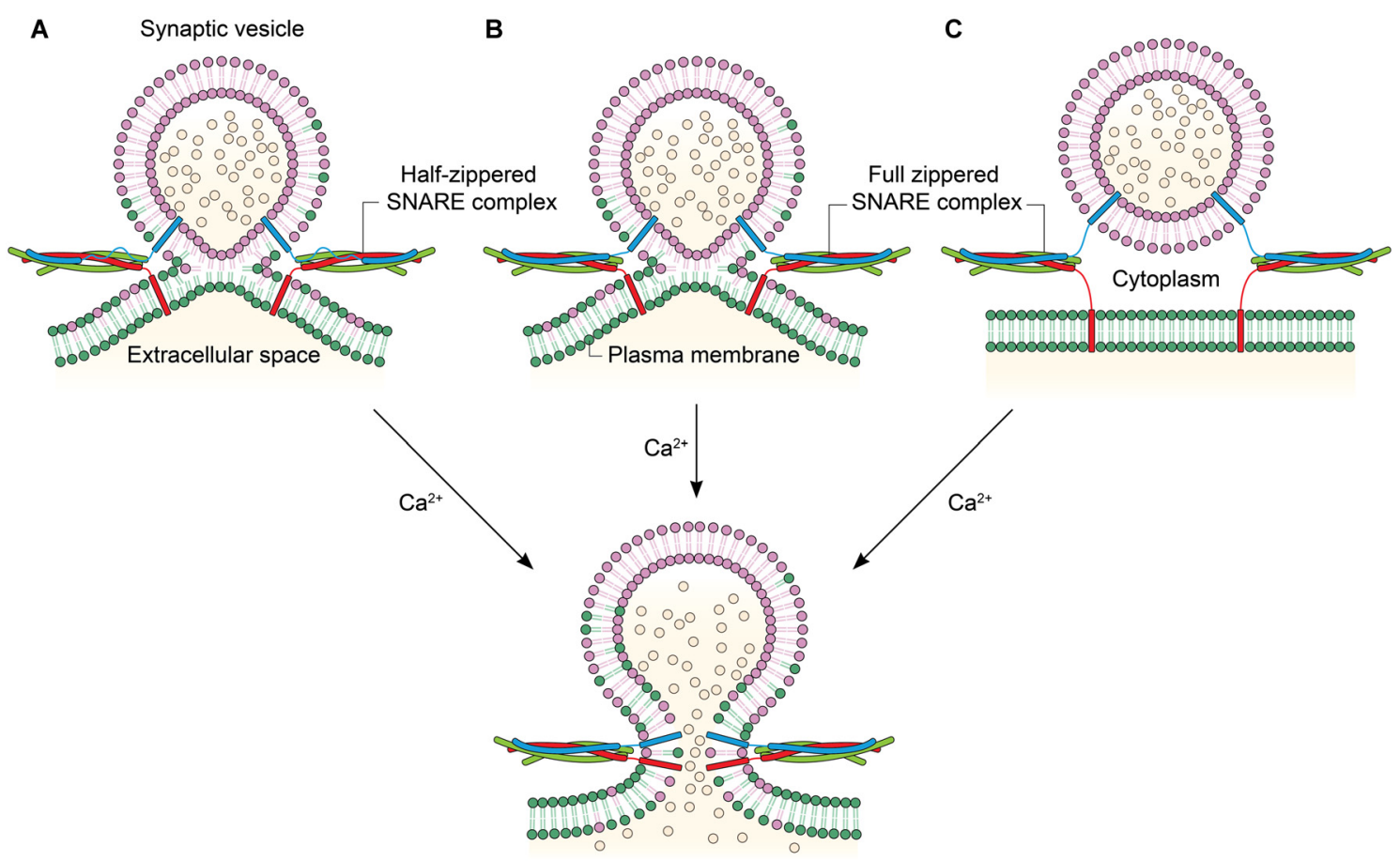

FIGURE 2 | Linking the degree of zippering to the hemifusion. (A) Hemifusion is achieved by only the zippering of the N-terminal half of SNARE motifs. Ca ${ }^{2+}$ induces fusion pore opening by enabling full SNARE zippering through the C-terminal half of SNARE motifs and TMDs. (B) Full SNARE zippering induces hemifusion. A fusion pore can be opened by $\mathrm{Ca}^{2+}$. (C) Full SNARE zippering is required to dock vesicles to the plasma membrane. In this case, Ca ${ }^{2+}$ may drive hemifusion and subsequent full fusion almost simultaneously to achieve fast exocytosis.

\section{HEMIFUSION IN THE ENDOCYTOTIC PATHWAY}

In the endocytotic pathway, vesicles are created by membrane fission. Membrane fission is a topologically opposite process to membrane fusion. But, this reaction might as well transit through the hemifusion. A GTPase dynamin is the best-studied membrane fission protein. The dynamin protein binds to the neck of the spherical membrane sac bulged from the plasma membrane (Figure 3). The GTP-driven

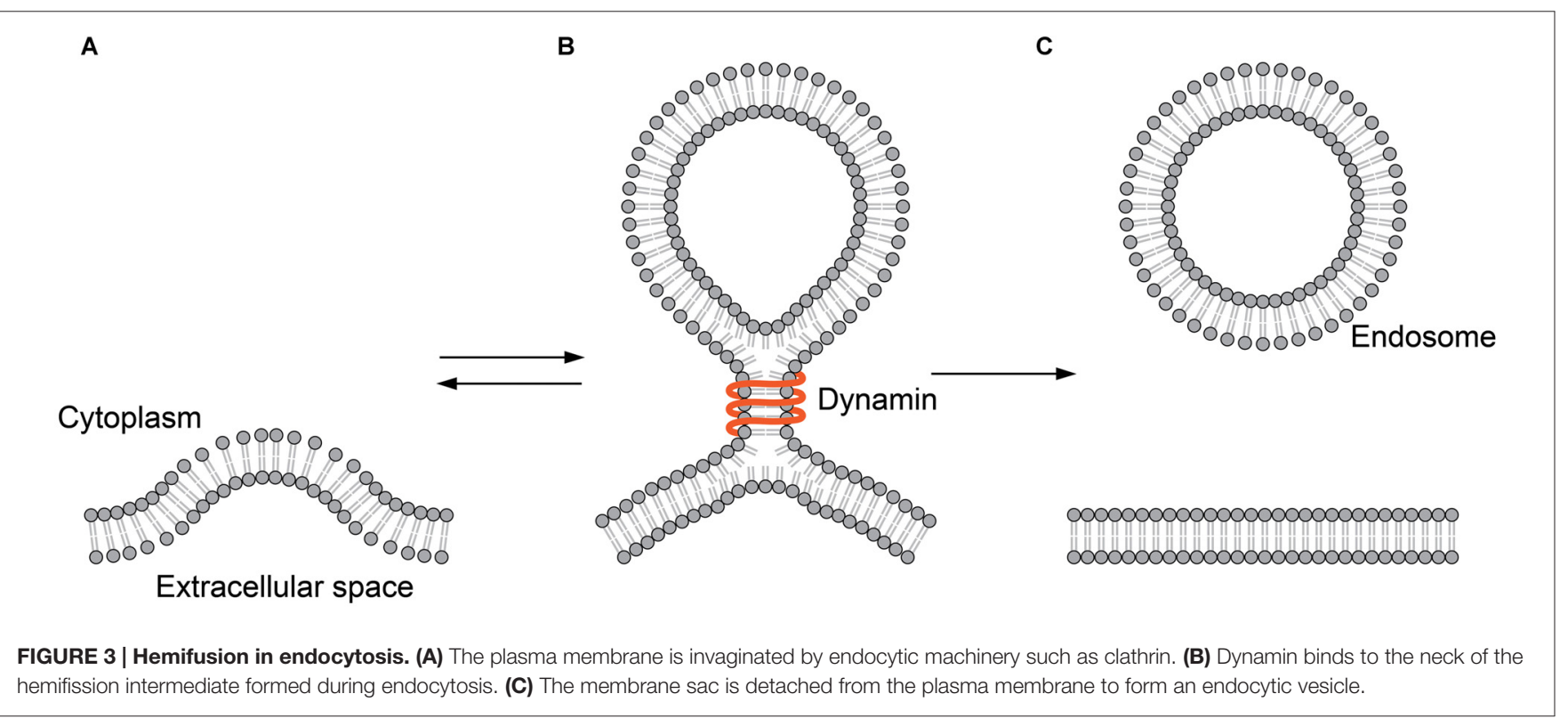


conformational change of dynamin constricts the neck to detach the vesicle from the plasma membrane (Antonny et al., 2016).

In a clever experiment using a tubular membrane capillary, Bashkirov et al. (2008) have shown that dynamin-induced membrane fission is leak-free, representing the fission through hemifusion (Bashkirov et al., 2008). Here, it is clear that dynamins remain on the periphery and are not integral parts of the intermediate. Thus, the role of the protein is clearly defined as the mechanical energy source that drives the remodeling of the membrane.

Recently, hemifusion and hemifission structures were observed in live cells, which provided strong evidence of the hemifusion model against that of a TMD-lined pore. Zhao et al. (2016) observed membrane fusion directly in live chromaffin cells in real time using super-resolution stimulated emission depletion microscopy. They observed a $\Omega$-shaped hemifusion structure in the live cell, adding further evidence that the hemifusion indeed exists along both the fusion and fission pathways.

\section{PERSPECTIVES}

Although exocytotic membrane fusion was initially considered to traverse the TMD-lined fusion pore, evidence has accumulated to support an alternative pathway through the lipidic hemifusion

\section{REFERENCES}

Albillos, A., Dernick, G., Horstmann, H., Almers, W., Alvarez de Toledo, G., and Lindau, M. (1997). The exocytotic event in chromaffin cells revealed by patch amperometry. Nature 389, 509-512. doi: 10.1038/ 39081

Alvarez de Toledo, G., Fernández-Chacón, R., and Fernández, J. M. (1993). Release of secretory products during transient vesicle fusion. Nature 363, 554-558. doi: 10.1038/363554a0

Antonny, B., Burd, C., De Camilli, P., Chen, E., Daumke, O., Faelber, K., et al. (2016). Membrane fission by dynamin: what we know and what we need to know. EMBO J. 35, 2270-2284. doi: 10.15252/embj. 201694613

Bao, H., Goldschen-Ohm, M., Jeggle, P., Chanda, B., Edwardson, J. M., and Chapman, E. R. (2016). Exocytotic fusion pores are composed of both lipids and proteins. Nat. Struct. Mol. Biol. 23, 67-73. doi: 10.1038/nsmb.3141

Bashkirov, P. V., Akimov, S. A., Evseev, A. I., Schmid, S. L., Zimmerberg, J., and Frolov, V. A. (2008). GTPase cycle of dynamin is coupled to membrane squeeze and release, leading to spontaneous fission. Cell 135, 1276-1286. doi: 10.1016/j. cell.2008.11.028

Breckenridge, L. J., and Almers, W. (1987). Currents through the fusion pore that forms during exocytosis of a secretory vesicle. Nature 328, 814-817. doi: $10.1038 / 328814 \mathrm{a} 0$

Chang, C. W., Chiang, C. W., Gaffaney, J. D., Chapman, E. R., and Jackson, M. B. (2016). Lipid-anchored synaptobrevin provides little or no support for exocytosis or liposome fusion. J. Biol. Chem. 291, 2848-2857. doi: 10.1074/jbc. M115.701169

Chanturiya, A., Chernomordik, L. V., and Zimmerberg, J. (1997). Flickering fusion pores comparable with initial exocytotic pores occur in protein-free phospholipid bilayers. Proc. Natl. Acad. Sci. U S A 94, 14423-14428. doi: 10.1073/pnas.94.26.14423

Chen, Y. A., Scales, S. J., and Scheller, R. H. (2001). Sequential SNARE assembly underlies priming and triggering of exocytosis. Neuron 30, 161-170. doi: 10.1016/s0896-6273(01)00270-7 and fusion pore. The fusion pathway through the hemifusion appears now to be shared by many membrane fusion systems including viral-cell and intracellular membrane fusion. Not surprisingly, the common hemifusion intermediate is shared by endocytotic membrane fission as well, where no TMD is directly involved in the process. The core feature of the hemifusion is that it is lipidic in nature, although some regulatory participation of TMDs cannot be ruled out. In this model, the proteins may stay at the periphery, corralling lipids at the fusion center to undergo merging. As cryo EM and other imaging methods are making significant strides in improving resolution, we are sure that the mechanistic models described here will be tested in the very near future.

\section{AUTHOR CONTRIBUTIONS}

D-HK, BK and Y-KS wrote the article. All authors reviewed the manuscript.

\section{FUNDING}

This work was supported by the National Institutes of Health GM05290 and 5U54GM087519. Korea Healthcare Technology R\&D Project, Ministry of Health and Welfare, Republic of Korea (Grant No.: HN14C01010000).

Chernomordik, L. V., and Kozlov, M. M. (2003). Protein-lipid interplay in fusion and fission of biological membranes. Annu. Rev. Biochem. 72, 175-207. doi: 10.1146/annurev.biochem.72.121801.161504

Chernomordik, L. V., and Kozlov, M. M. (2008). Mechanics of membrane fusion. Nat. Struct. Mol. Biol. 15, 675-683. doi: 10.1038/nsmb.1455

Chernomordik, L. V., Frolov, V. A., Leikina, E., Bronk, P., and Zimmerberg, J. (1998). The pathway of membrane fusion catalyzed by influenza hemagglutinin: restriction of lipids, hemifusion, and lipidic fusion pore formation. J. Cell Biol. 140, 1369-1382. doi: 10.1083/jcb.140.6.1369

Chernomordik, L. V., Vogel, S. S., Sokoloff, A., Onaran, H. O., Leikina, E. A. and Zimmerberg, J. (1993). Lysolipids reversibly inhibit $\mathrm{Ca}^{2+}$, GTP- and $\mathrm{pH}$-dependent fusion of biological membranes. FEBS Lett. 318, 71-76. doi: 10.1016/0014-5793(93)81330-3

Chow, R. H., von Rüden, L., and Neher, E. (1992). Delay in vesicle fusion revealed by electrochemical monitoring of single secretory events in adrenal chromaffin cells. Nature 356, 60-63. doi: 10.1038/356060a0

Dhara, M., Yarzagaray, A., Makke, M., Schindeldecker, B., Schwarz, Y., Shaaban, A., et al. (2016). v-SNARE transmembrane domains function as catalysts for vesicle fusion. Elife 5:e17571. doi: 10.7554/eLife.17571

Diao, J., Grob, P., Cipriano, D. J., Kyoung, M., Zhang, Y., Shah, S., et al. (2012). Synaptic proteins promote calcium-triggered fast transition from point contact to full fusion. Elife 1:e00109. doi: 10.7554/eLife.00109

Ellena, J. F., Liang, B., Wiktor, M., Stein, A., Cafiso, D. S., Jahn, R. et al. (2009). Dynamic structure of lipid-bound synaptobrevin suggests a nucleation-propagation mechanism for trans-SNARE complex formation. Proc. Natl. Acad. Sci. U S A 106, 20306-20311. doi: 10.1073/pnas.09083 17106

Fang, Q., Berberian, K., Gong, L. W., Hafez, I., Sorensen, J. B., and Lindau, M (2008). The role of the C terminus of the SNARE protein SNAP-25 in fusion pore opening and a model for fusion pore mechanics. Proc. Natl. Acad. Sci. U S A 105, 15388-15392. doi: 10.1073/pnas.0805377105

Fiebig, K. M., Rice, L. M., Pollock, E., and Brunger, A. T. (1999). Folding intermediates of SNARE complex assembly. Nat. Struct. Biol. 6, 117-123. doi: $10.1038 / 5803$ 
Gao, Y., Zorman, S., Gundersen, G., Xi, Z., Ma, L., Sirinakis, G., et al. (2012). Single reconstituted neuronal SNARE complexes zipper in three distinct stages. Science 337, 1340-1343. doi: 10.1126/science. 1224492

Giraudo, C. G., Eng, W. S., Melia, T. J., and Rothman, J. E. (2006). A clamping mechanism involved in SNARE-dependent exocytosis. Science 313, 676-680. doi: 10.1126/science. 1129450

Han, X., Wang, C. T., Bai, J., Chapman, E. R., and Jackson, M. B. (2004). Transmembrane segments of syntaxin line the fusion pore of $\mathrm{Ca}^{2+}$-triggered exocytosis. Science 304, 289-292. doi: 10.1126/science.1095801

Heo, P., Yang, Y., Han, K. Y., Kong, B., Shin, J. H., Jung, Y., et al. (2016). A chemical controller of SNARE-driven membrane fusion that primes vesicles for $\mathrm{Ca}^{2+}$-Triggered millisecond exocytosis. J. Am. Chem. Soc. 138, 4512-4521. doi: 10.1021/jacs.5b13449

Hernandez, J. M., Stein, A., Behrmann, E., Riedel, D., Cypionka, A., Farsi, Z., et al. (2012). Membrane fusion intermediates via directional and full assembly of the SNARE complex. Science 336, 1581-1584. doi: 10.1126/science.1221976

Hui, E., Johnson, C. P., Yao, J., Dunning, F. M., and Chapman, E. R. (2009). Synaptotagmin-mediated bending of the target membrane is a critical step in $\mathrm{Ca}^{2+}$-regulated fusion. Cell 138, 709-721. doi: 10.1016/j.cell.2009.05.049

Jun, Y., and Wickner, W. (2007). Assays of vacuole fusion resolve the stages of docking, lipid mixing, and content mixing. Proc. Natl. Acad. Sci. U S A 104, 13010-13015. doi: 10.1073/pnas.0700970104

Kemble, G. W., Danieli, T., and White, J. M. (1994). Lipid-anchored influenza hemagglutinin promotes hemifusion, not complete fusion. Cell 76, 383-391. doi: 10.1016/0092-8674(94)90344-1

Klyachko, V. A., and Jackson, M. B. (2002). Capacitance steps and fusion pores of small and large-dense-core vesicles in nerve terminals. Nature 418, 89-92. doi: 10.1038/nature00852

Kozlov, M. M., Chernyi, V. V., Sokolov, V. S., Ermakov Iu, A., and Markin, V. S. (1983). Theory of hydrophobic ion adsorption in bilayer lipid membranes taking into account their lateral interaction and charge discreteness. Biofizika 28, 61-66.

Lang, T., Bruns, D., Wenzel, D., Riedel, D., Holroyd, P., Thiele, C., et al. (2001). SNAREs are concentrated in cholesterol-dependent clusters that define docking and fusion sites for exocytosis. EMBO J. 20, 2202-2213. doi: 10.1093/emboj/20.9.2202

Li, F., Pincet, F., Perez, E., Eng, W. S., Melia, T. J., Rothman, J. E., et al. (2007). Energetics and dynamics of SNAREpin folding across lipid bilayers. Nat. Struct. Mol. Biol. 14, 890-896. doi: 10.1038/nsmb1310

Lollike, K., Borregaard, N., and Lindau, M. (1995). The exocytotic fusion pore of small granules has a conductance similar to an ion channel. J. Cell Biol. 129, 99-104. doi: 10.1083/jcb.129.1.99

Lou, X., and Shin, Y. K. (2016). SNARE zippering. Biosci. Rep. 36:e00327. doi: 10.1042/BSR20160004

Lu, X., Zhang, F., McNew, J. A., and Shin, Y. K. (2005). Membrane fusion induced by neuronal SNAREs transits through hemifusion. J. Biol. Chem. 280, 30538-30541. doi: 10.1074/jbc.M506862200

Martens, S., Kozlov, M. M., and McMahon, H. T. (2007). How synaptotagmin promotes membrane fusion. Science 316, 1205-1208. doi: 10.1126/science. 1142614

Matos, M. F., Mukherjee, K., Chen, X., Rizo, J., and Sudhof, T. C. (2003). Evidence for SNARE zippering during $\mathrm{Ca}^{2+}$-triggered exocytosis in PC12 cells. Neuropharmacology 45, 777-786. doi: 10.1016/s0028-3908(03)00318-6

McNew, J. A., Weber, T., Parlati, F., Johnston, R. J., Melia, T. J., Söllner, T. H., et al. (2000). Close is not enough: SNARE-dependent membrane fusion requires an active mechanism that transduces force to membrane anchors. J. Cell Biol. 150, 105-117. doi: 10.1083/jcb.150.1.105

Melia, T. J., Weber, T., McNew, J. A., Fisher, L. E., Johnston, R. J., Parlati, F., et al. (2002). Regulation of membrane fusion by the membrane-proximal coil of the t-SNARE during zippering of SNAREpins. J. Cell Biol. 158, 929-940. doi: $10.1083 /$ jcb.200112081

Melikyan, G. B., White, J. M., and Cohen, F. S. (1995). GPI-anchored influenza hemagglutinin induces hemifusion to both red blood cell and planar bilayer membranes. J. Cell Biol. 131, 679-691. doi: 10.1083/jcb.131.3.679

Min, D., Kim, K., Hyeon, C., Cho, Y. H., Shin, Y. K., and Yoon, T. Y. (2013). Mechanical unzipping and rezipping of a single SNARE complex reveals hysteresis as a force-generating mechanism. Nat. Commun. 4:1705. doi: $10.1038 /$ ncomms 2692
Neher, E., and Marty, A. (1982). Discrete changes of cell membrane capacitance observed under conditions of enhanced secretion in bovine adrenal chromaffin cells. Proc. Natl. Acad. Sci. U S A 79, 6712-6716. doi: 10.1073/pnas.79.21.6712

Oelkers, M., Witt, H., Halder, P., Jahn, R., and Janshoff, A. (2016). SNAREmediated membrane fusion trajectories derived from force-clamp experiments. Proc. Natl. Acad. Sci. U S A 113, 13051-13056. doi: 10.1073/pnas.1615885113

Poirier, M. A., Xiao, W., Macosko, J. C., Chan, C., Shin, Y. K., and Bennett, M. K. (1998). The synaptic SNARE complex is a parallel four-stranded helical bundle. Nat. Struct. Biol. 5, 765-769. doi: 10.1038/1799

Risselada, H. J., Kutzner, C., and Grubmuller, H. (2011). Caught in the act: visualization of SNARE-mediated fusion events in molecular detail. Chembiochem 12, 1049-1055. doi: 10.1002/cbic.201100020

Schaub, J. R., Lu, X., Doneske, B., Shin, Y. K., and McNew, J. A. (2006). Hemifusion arrest by complexin is relieved by $\mathrm{Ca}^{2+}$-synaptotagmin I. Nat. Struct. Mol. Biol. 13, 748-750. doi: 10.1038/nsmb1124

Shi, L., Shen, Q. T., Kiel, A., Wang, J., Wang, H. W., Melia, T. J., et al. (2012). SNARE proteins: one to fuse and three to keep the nascent fusion pore open. Science 335, 1355-1359. doi: 10.1126/science.1214984

Shin, J., Lou, X., Kweon, D. H., and Shin, Y. K. (2014). Multiple conformations of a single SNAREpin between two nanodisc membranes reveal diverse pre-fusion states. Biochem J. 459, 95-102. doi: 10.1042/BJ20131668

Söllner, T., Bennett, M. K., Whiteheart, S. W., Scheller, R. H., and Rothman, J. E. (1993a). A protein assembly-disassembly pathway in vitro that may correspond to sequential steps of synaptic vesicle docking, activation, and fusion. Cell 75, 409-418. doi: 10.1016/0092-8674(93)90376-2

Söllner, T., Whiteheart, S. W., Brunner, M., Erdjument-Bromage, H., Geromanos, S., Tempst, P., et al. (1993b). SNAP receptors implicated in vesicle targeting and fusion. Nature 362, 318-324. doi: 10.1038/362318a0

Sorensen, J. B., Wiederhold, K., Müller, E. M., Milosevic, I., Nagy, G., de Groot, B. L., et al. (2006). Sequential N- to C-terminal SNARE complex assembly drives priming and fusion of secretory vesicles. EMBO J. 25, 955-966. doi: 10.1038/sj.emboj.7601003

Su, Z., Ishitsuka, Y., Ha, T., and Shin, Y. K. (2008). The SNARE complex from yeast is partially unstructured on the membrane. Structure 16, 1138-1146. doi: 10.1016/j.str.2008.03.018

Sutton, R. B., Fasshauer, D., Jahn, R., and Brunger, A. T. (1998). Crystal structure of a SNARE complex involved in synaptic exocytosis at 2.4 A resolution. Nature 395, 347-353. doi: 10.1038/26412

Takamori, S., Holt, M., Stenius, K., Lemke, E. A., Grønborg, M., Riedel, D., et al. (2006). Molecular anatomy of a trafficking organelle. Cell 127, 831-846. doi: $10.1016 /$ j.cell.2006.10.030

Tong, J., Borbat, P. P., Freed, J. H., and Shin, Y. K. (2009). A scissors mechanism for stimulation of SNARE-mediated lipid mixing by cholesterol. Proc. Natl. Acad. Sci. U S A 106, 5141-5146. doi: 10.1073/pnas.0813138106

van den Bogaart, G., Holt, M. G., Bunt, G., Riedel, D., Wouters, F. S., and Jahn, R. (2010). One SNARE complex is sufficient for membrane fusion. Nat. Struct. Mol. Biol. 17, 358-364. doi: 10.1038/nsmb.1748

Weber, T., Zemelman, B. V., McNew, J. A., Westermann, B., Gmachl, M., Parlati, F., et al. (1998). SNAREpins: minimal machinery for membrane fusion. Cell 92, 759-772. doi: 10.1016/s0092-8674(00)81404-x

Williams, D., Vicôgne, J., Zaitseva, I., McLaughlin, S., and Pessin, J. E. (2009). Evidence that electrostatic interactions between vesicle-associated membrane protein 2 and acidic phospholipids may modulate the fusion of transport vesicles with the plasma membrane. Mol. Biol. Cell 20, 4910-4919. doi: 10.1091/mbc.E09-04-0284

Wong, J. L., Koppel, D. E., Cowan, A. E., and Wessel, G. M. (2007). Membrane hemifusion is a stable intermediate of exocytosis. Dev. Cell 12, 653-659. doi: 10.1016/j.devcel.2007.02.007

Xu, Y., Zhang, F., Su, Z., McNew, J. A., and Shin, Y. K. (2005). Hemifusion in SNARE-mediated membrane fusion. Nat. Struct. Mol. Biol. 12, 417-422. doi: 10.1038/nsmb921

Xu, H., Zick, M., Wickner, W. T., and Jun, Y. (2011). A lipid-anchored SNARE supports membrane fusion. Proc. Natl. Acad. Sci. U S A 108, 17325-17330. doi: $10.1073 /$ pnas.1113888108

Yang, L., and Huang, H. W. (2002). Observation of a membrane fusion intermediate structure. Science 297, 1877-1879. doi: 10.1126/science.1074354

Yang, Y., Shin, J. Y., Oh, J.-M., Jung, C. H., Hwang, Y., Kim, S., et al. (2010). Dissection of SNARE-driven membrane fusion and neuroexocytosis by 
wedging small hydrophobic molecules into the SNARE zipper. Proc. Natl. Acad. Sci. U S A 107, 22145-22150. doi: 10.1073/pnas.1006899108

Yoon, T. Y., Okumus, B., Zhang, F., Shin, Y. K., and Ha, T. (2006). Multiple intermediates in SNARE-induced membrane fusion. Proc. Natl. Acad. Sci. U S A 103, 19731-19736. doi: 10.1073/pnas.06060 32103

Zampighi, G. A., Zampighi, L. M., Fain, N., Lanzavecchia, S., Simon, S. A., and Wright, E. M. (2006). Conical electron tomography of a chemical synapse: vesicles docked to the active zone are hemi-fused. Biophys. J. 91, 2910-2918. doi: 10.1529/biophysj.106.084814

Zhao, W. D., Hamid, E., Shin, W., Wen, P. J., Krystofiak, E. S., Villarreal, S. A., et al. (2016). Hemi-fused structure mediates and controls fusion and fission in live cells. Nature 534, 548-552. doi: 10.1038/nature 18598

Zhou, P., Bacaj, T., Yang, X., Pang, Z. P., and Südhof, T. C. (2013). Lipidanchored SNAREs lacking transmembrane regions fully support membrane fusion during neurotransmitter release. Neuron 80, 470-483. doi: 10.1016/j. neuron.2013.09.010

Zorman, S., Rebane, A. A., Ma, L., Yang, G., Molski, M. A., Coleman, J., et al. (2014). Common intermediates and kinetics, but different energetics, in the assembly of SNARE proteins. Elife 3:e03348. doi: 10.7554/eLife.03348

Conflict of Interest Statement: The authors declare that the research was conducted in the absence of any commercial or financial relationships that could be construed as a potential conflict of interest.

Copyright (๑) 2017 Kweon, Kong and Shin. This is an open-access article distributed under the terms of the Creative Commons Attribution License (CC BY). The use, distribution and reproduction in other forums is permitted, provided the original author(s) or licensor are credited and that the original publication in this journal is cited, in accordance with accepted academic practice. No use, distribution or reproduction is permitted which does not comply with these terms. 